\author{
Ferdinando Maggioni \\ Silvia Ruffatti \\ Federica Viaro \\ Federico Mainardi \\ Carlo Lisotto \\ Giorgio Zanchin
}

\section{A case of posterior scleritis: differential diagnosis of ocular pain}

Received: 13 January 2007

Accepted in revised form: 13 February 2007

Published online: 11 May 2007

F. Maggioni (四) • S. Ruffatti • F. Viaro

G. Zanchin

Headache Centre,

Department of Neurosciences,

University of Padua,

Via Giustiniani 5, I-35128 Padova, Italy

e-mail: ferdinando.maggioni@unipd.it

Tel.: +39-049-8213600

Fax: +39-049-8218568

F. Mainardi

Headache Centre,

SS. Giovanni e Paolo Hospital,

Venice, Italy

C. Lisotto

Headache Centre,

S. Vito al Tagliamento Hospital,

Pordenone, Italy

\begin{abstract}
Posterior scleritis is a rare cause of ocular pain, due to scleral inflammation, presenting with periocular pain, pain on movement and decreased vision. Although anterior scleritis may be associated with this condition, ocular signs may be absent. We report a case of posterior scleritis, presenting with right-sided ocular and periocular pain, exacerbated by ocular movements, irradiating to the ipsilateral temple and zygoma, not associated with visual disturbances at onset. Diagnosis was made with ultrasonography and confirmed by brain and orbital MRI. Differential diagnosis of facial pain, in particular, affecting the periorbital region, is discussed.
\end{abstract}

In the presence of ocular pain, even in the absence of ocular signs, an ophthalmologic consultation should be performed.

Keywords Posterior scleritis • Facial pain $\cdot$ Differential diagnosis

\section{Introduction}

Posterior scleritis is an uncommon form of scleral inflammation affecting the sclera behind the ora serrata and is involved in changes in adjacent structures in the posterior segment of the eye (choroid, retina and optic nerve) and the anterior segment $[1,2]$. It can be confused with several inflammatory and non-inflammatory ocular diseases. If presenting as a nodular mass it can be mistaken for an ocular tumour [3]. It is therefore often under-recognised. Anterior scleritis can be associated with this condition. Posterior scleritis can be idiopathic or, in nearly
$40 \%-50 \%$ of cases, associated with systemic disorders, which include rheumatic diseases, systemic vasculitis, and autoimmune disease; infections account for less than $10 \%$ of these $[4,5]$. It most frequently occurs in middle-aged women, when it is often associated with connective tissue disorders, while the idiopathic form is more frequent in young patients [3]. Women are more frequently affected than men [5]. Periocular pain, pain on movement and decreased vision are the most frequent symptoms. Redness of the affected eye can be observed. Ocular complications associated with posterior scleritis may involve both the anterior and posterior segment of the eye and include anterior uveitis, increased intraocular pressure, 

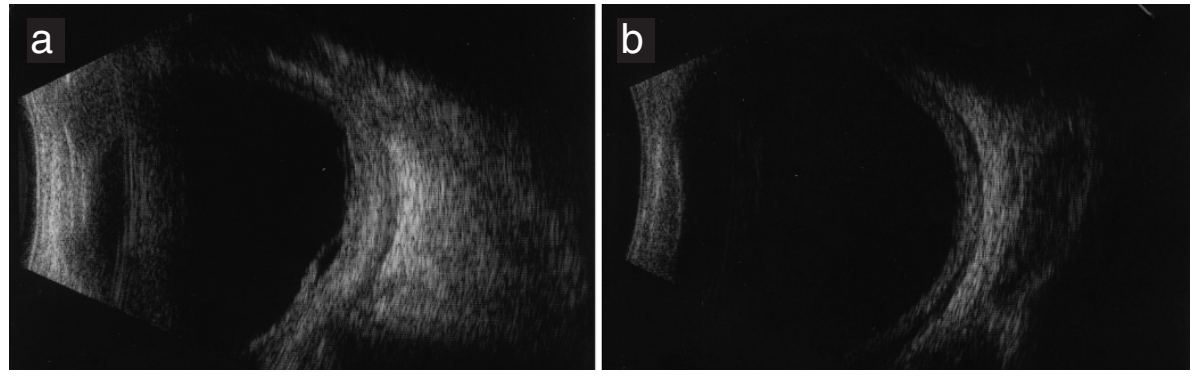

Fig. 1 B-mode ultrasound of the right eye. (a) On admittance: posterior scleral thickening and exudative retinal detachment. (b) After one month: reduction of scleral thickening, fluid in the Tenon capsule and regression of retinal detachment

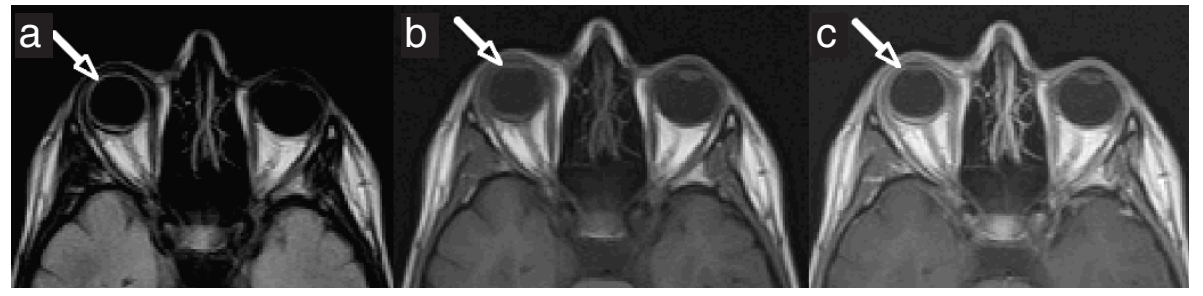

Fig. 2 Brain and orbit MRI with and without gadolinium: hyperintensity around the right ocular globe, more evident after gadolinium. (a) Flair; (b) and (c) T1-weighted images without and with gadolinium

vitritis, macular oedema and exudative retinal detachment [5]. In up to $50 \%$ of cases it can be bilateral [5]. Ultrasonography is the key investigation necessary to make a diagnosis of posterior scleritis [4]. Brain CT scan, MRI and fundus fluorescein angiography can also be used. Posterior scleritis responds to therapy with systemic nonsteroidal anti-inflammatory drugs, corticosteroids and immunosuppressive drugs [4]. In the International Headache Society Classification [ICHD-2] [6] it is included in the chapter "Headache attributed to ocular inflammatory disorder", although not specifically mentioned (11.3.4). We report a case of a 26-year-old woman who complained of right-sided ocular pain at onset, not associated with visual disturbances. The diagnosis of ocular pain, especially if not associated with ocular signs or visual loss, may be a challenge for the clinician. Differential diagnosis of ocular pain is discussed.

\section{Case history}

A 26-year-old woman began to complain of right-sided ocular and periocular pain, exacerbated by ocular movements, irradiating to the ipsilateral temple and zygoma, not associated with visual disturbances. Admitted to our Emergency Department, she underwent an ophthalmologic consultation, which showed normal visual acuity and fundus oculi examination. No ocular signs were detected. Trigeminal neuralgia was suspected and she was then treated unsuccessfully with analgesics. Seven days later she underwent a second ophthalmologic and a neurological consultation, which were both normal. After one week, about 15 days after the onset of symptoms, she experi- enced a worsening of facial pain, which became intolerable. She also developed conjunctival injection, eyelid oedema and referred the vision of a central scotoma. She was then admitted to our clinic. On admission, fundus oculi examination revealed in the right eye a hyperaemic optic disc with blurred margins, while the left eye was normal. The patient referred blurred vision in the right eye with a central scotoma. The remaining neurological examination was normal. A third ophthalmologic consultation showed a visual acuity of 20/200 in the right eye (20/20 in the left) with conjunctival injection. Fundus oculi confirmed the above reported findings and, in addition, some retinal folds were detected. Ultrasound was performed, which revealed posterior scleral thickening and exudative retinal detachment, suggesting posterior scleritis (Fig. 1). Routine blood tests were normal, as were immunological exams aimed at ruling out rheumatic disease (complement fractions $\mathrm{C} 3$ and $\mathrm{C} 4$, rheumatoid factor, $\operatorname{IgG}, \operatorname{Ig} \mathrm{A}, \operatorname{IgM}$, circulating immunocomplexes [CIC], antinuclear antibodies, antineutrophil cytoplasmic antibodies and antiphospholipid antibodies). Cerebrospinal fluid examination, performed to rule out inflammatory and infectious disease, was normal, as was a chest X-ray. Serologic tests for herpes simplex, herpes zoster, syphilis, Lyme disease, HIV and toxoplasma were negative. Brain and orbital MRI with and without gadolinium enhancement was at first considered normal; re-evaluation, performed after the diagnosis of posterior scleritis was made, showed hyperintensity around the right ocular globe representing the inflammatory reaction, more evident after gadolinium enhancement (Fig. 2). The patient was treated with methylprednisolone i.v. $1 \mathrm{~g} /$ day for 10 days with complete regression of pain and clear improvement of visual disturbances. An ophthalmologic examination, performed 10 
days after the end of treatment, showed a visual acuity of $20 / 120$ in the right eye, which completely normalised after 40 days. Fundus oculi examination still showed some retinal folds; the optic disc was normal. Ultrasonography showed a reduction of scleral thickening, fluid in the Tenon capsule and regression of retinal detachment (Fig. 1). The exam performed in the left eye was normal. A month after discharge the patient began to complain of left periocular pain, without visual loss or eyelid oedema. Ultrasonography showed scleral thickening and fluid in the Tenon capsule in the left eye, suggesting posterior scleritis; she fully recovered after a further course of steroid treatment. An ophthalmologic examination performed after four months showed a normalisation of ultrasonographic findings and normal visual acuity in both eyes.

\section{Discussion}

Different conditions may present with facial pain, and defining its aetiology can be challenging for the clinician. It is important to be aware of the rare conditions that can cause facial pain in order to make an accurate diagnosis. Posterior scleritis is a rare cause of facial pain, mostly periocular, but possibly referred to the forehead, zygoma or temple, usually described as penetrating in character and often exacerbated by movement. It should be suspected in patients who present with ocular pain in addition to visual disturbances or ocular signs. Visual loss is the most important complication of posterior scleritis, but is not always present; redness of the affected eye can be observed. Proptosis, eyelid oedema and restriction of ocular movements can be seen with intense periscleral inflammation spreading to orbital and extraocular muscles [7]. It is one of the most commonly misdiagnosed conditions in ophthalmology, especially when, as in our case, pain is not associated with visual disturbances or ocular signs at the onset of symptoms and may prompt a central nervous system evaluation until their development. Due to its variable presentation, posterior scleritis can be confused with a number of different entities, which can cause facial pain. Among diseases affecting the eye, orbital pseudotumour and tumours (primary or secondary) should be considered. In these cases ocular signs, such as conjunctival injection, proptosis, eyelid oedema and chemosis are usually more evident and ophthalmoplegia can be associated [8]. Vascular diseases may also cause ocular pain. Intracavernous carotid artery aneurysm and carotid cavernous fistula present with ocular pain, usually in association with ophthalmoplegia and, especially in the latter case, marked orbital signs. Carotid artery dissection may present with facial or ocular pain frequently, but not necessarily, in association with Claude-Bernard-Horner syndrome. Neoplastic, infectious and inflammatory diseases as well as Tolosa Hunt syndrome, which affect the cavernous sinus, may present with painful ophthalmoplegia, even though at onset ocular movements may be normal [8]. Trigeminal neuralgia is another important cause of facial pain, stabbing in character, which can involve only the first trigeminal branch and therefore present with periocular pain, although this localisation is the least frequent (5\%) [9]. In the case described above this was the first diagnosis made by the neurologist when ocular signs were still not present. Giant cell arteritis, mostly affecting middle-aged women, is characterised by pain affecting the temporal area, sometimes spreading to frontal, orbital or occipital regions. If this condition is suspected it is important to look for tenderness and decreased pulsation of the temporal artery, increased erythrocyte sedimentation rate and jaw claudication. Permanent partial or complete loss of vision in one or both eyes occurs in up to $20 \%$ of patients and is often an early manifestation of the disease [10]. In young patients presenting with ocular pain, exacerbated by movement and in association with loss of vision, optic neuritis should be suspected. In this condition fundus oculi examination is often normal. In the case described, lumbar puncture had been performed to rule out demyelinating disease. Lastly, glaucoma should be considered as a cause of ocular pain. Acute angle-closure glaucoma is characterised by periorbital pain, conjunctival reddening, lacrimation, mydriasis and blurring of vision and can be easily identified [11]. More insidious is intermittent angle-closure glaucoma presenting with attacks of facial pain or headache, blurred vision, nausea and vomiting, in the absence of ocular signs such as conjunctival injections or pupillary abnormalities. The eye is usually white and normal in appearance. In this case gonioscopy is essential to the diagnosis $[9,11]$. Many different diseases may present with ocular pain. For a correct diagnosis it is therefore very important to perform an accurate neurological and general examination, including fundus oculi, searching for associated systemic and ocular signs. An ophthalmologic consultation or ultrasonography should be considered, especially in the presence of fundus oculi abnormalities. Neuroimaging, in particular MRI, could help in the diagnostic process, pointing out to the radiologist the clinical suspicion; as shown in our case, abnormalities seen in posterior scleritis may not be seen if not specifically searched for. If posterior scleritis is suspected, it is very important to make the diagnosis as soon as possible and start treatment promptly, in order to avoid permanent loss of vision. Strict follow-up is also very important, as recurrence or bilateral involvement, as seen in our patient, is frequent. 


\section{References}

1. Watson P, Hazleman BL, Pavésio C, Green WR (2004) Sclera and systemic disorders, 2nd edn. Oxford, Butterworth Heinemann

2. Sadiq Z, Shepherd R, Vardy S (2003) Posterior scleritis - an unusual presentation of facial pain. Br J Oral Maxillofac Surg 41:62-63

3. Osman Saatci A, Saatci I, Kocak N, Durak I (2001) Magnetic resonance imaging characteristics of posterior scleritis mimicking choroidal mass. Eur J Radiol 39:88-91

4. McCluskey PJ, Watson PG, Lightman S et al (1999) Posterior scleritis: clinical features, systemic associations, and outcome in a large series of patients. Ophthalmology 106:2380-2386
5. Jabs DA, Mudun A, Dunn JP, Marsh MJ (2000) Episcleritis and scleritis: clinical features and treatment results. Am J Ophthalmol 130:469-476

6. Headache Classification Subcommittee of the International Headache Society. (2004) The International Classification of Headache Disorders, 2nd edn. Cephalalgia 24[Suppl 1]:9-160

7. Biswas J, Mittal S, Ganesh SK et al (1998) Posterior scleritis: clinical profile and imaging characteristics. Indian J Ophthalmol 46:195-202

8. Gladstone JP, Dodick DW (2004) Painful ophthalmoplegias: overview with focus on Tolosa-Hunt Syndrome. Curr Pain Headache Rep 8:321-329
9. Brazis PW, Lee AJ, Stewart M, Capobianco D (2002) Clinical review: the differential diagnosis of pain in the quiet eye. Neurologist 8:82-100

10. Salvarani C, Cantini F, Boiardi L, Hunder GG (2002) Polymyalgia rheumatica and giant-cell arteritis. $\mathrm{N}$ Engl J Med 347:261-271

11. Maggioni F, Dainese F, Mainardi F et al (2005) Intermittent angle-closure glaucoma in the presence of a white eye, posing as retinal migraine. Cephalalgia 25:622-626 